\title{
A Few Words about Food Science and Nutrition and its Impact on Aging and Age-Associated Diseases
}

ISSN: 2640-9208

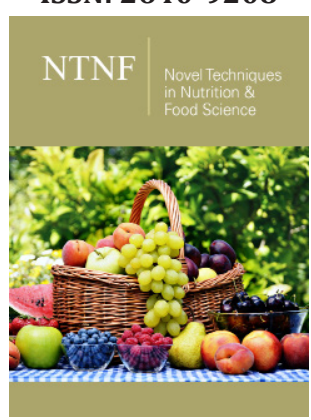

${ }^{* 1}$ Corresponding author: Pradip Kumar Das, Associate Professor, India

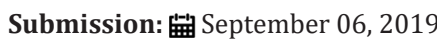

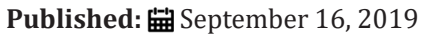

Volume 4 - Issue 3

How to cite this article: Pradip Kumar Das. A Few Words about Food Science and Nutrition and its Impact on Aging and AgeAssociated Diseases. Nov Tech Nutri Food Sci. 4(3).NTNF.000589.2019.

DOI: 10.31031/NTNF.2019.04.000589

Copyright@ Pradip Kumar Das, This article is distributed under the terms of the Creative Commons Attribution 4.0 International License, which permits unrestricted use and redistribution provided that the original author and source are credited.

\author{
Pradip Kumar Das* \\ Associate Professor, India
}

\begin{abstract}
Food science and nutrition science are equally important and mutually dependent scientific disciplines. Due to demographic transition, older population has increased, and it has been observed that there has been a transition in terms of caregivers. Although there is uniformity in nature and habit, heterogeneity in terms of social, economic, physical and food frequency among the older is observed. Thus, it is necessary to study the food habit and nutritional status of the aged persons. Besides conceptual study, this paper highlights the impact of food science and nutrition on aging and age-associated diseases in general and in old Aged Homes in Purulia, India.
\end{abstract}

Keywords: Food science; Nutrition science; Nutritional status; Diet; Physical activity; Age-associated diseases; Aging

\section{Introduction}

Food science is the study of physical, biological and chemical make-up of food and the concepts underlying food processing. Nutrition science has evolved with the growing understanding of macronutrients and the physiology of energy with the discovery of essential nutrients-the vitamin era, the birth of functional foods and the concept of individualized nutrition. The American Medical Association Council defines food and nutrition as, "The science of food, the nutrients and other substances therein, their action, interaction and balance in relation to health and disease and the processes by which the organism ingests, digests, absorbs, transports, utilizes and excretes food substances." India, the second largest country in the world having 72 million older persons above 60 years of age as of 2001[1], the older in the age group 60 and above is expected to increase from 71 million in 2001 to 179 million in 2031. According to India Aging Report, 2017 by the United Nations Population Fund (UNFPA), the share of population over the age of 60 could increase from $8 \%$ in 2015 to $19 \%$ in 2050. This increasing proportion of older should have a direct impact on the demand for health services and pension and social security payments [2]. According to the report of the National Sample Survey Office (NSSO), about three-fourth of the older is supported by their own children, $7 \%$ and $11 \%$ of women were supported by their spouses and $8 \%$ and $12 \%$ were supported by institutional care. Another study observed $71 \%$ of the older live with their children (family care), $26.3 \%$ live by themselves and with spouse and about $2.73 \%$ live with institutional support [3]. Nutrition plays an important role in determining the health and well-being of the older [4]. In the present era, the young generation due to their fast life style having problems in coping up with special needs of the older and requiring resources for special care are not able to spare their time to older which had developed a situation for the need of Old Age Homes (OAH). This concept draws the attention of the present author to study food and nutrition science and their impact on aging and age- related diseases.

Objective of the study

$>\quad$ To make conceptual study of food and nutrition science.

$>$ To focus their impact on aging and age-associated diseases.

$>$ To assess nutritional status, food habits and physical activity of inmates above 60 years residing in Purulia and its adjacent areas.

$>$ To offer suggestions based on the above study. 


\section{Literature review}

In India, a limited study has been conducted on food and nutrition science and their impact on aged persons. OAH provides not only shelter but also provides nutrition and health care to the older. Health of the older population at large is determined by the economic and human development of the place where they reside. Similarly, facilities in $\mathrm{OAH}$, especially of healthcare vary with the amount paid by the inmates [5]. Studies have reported on the assessment of nutritional status of older under institutional care. It was observed that with the number of older living in any kind of 'institutional setting' had an impact on their nutrition status. Older faced weight loss, limited capacities and reduced functional capability which controls social network, health and functional status (WHO Nutrition for Older Persons, 2018). The studies conducted by Centre for Disease Control (CDC) and other groups have carried out substantial research for showing significant relationship between nutritional choice and health ailments like hyper insulin-anemia, hypertension, dyslipidemia, coronary heart disease and diabetes. This acknowledges nutrition now as an important aspect in determining the health status of the individual [6]. There is an urgency to study the health and nutrition care provided at $\mathrm{OAH}$. At $\mathrm{OAH}$, older population of healthy and malnutrition reside with physiological conditions such as diabetes, hypertension, obesity, hyperlipidemia, etc. [7]. At homes, a predecided menu for the whole week is served which is unplanned keeping in view neither the health or the nutritional status of the inmates nor with the advice of nutrition expert. The facilities provided vary according to the finance subscribed by the inmates at different OAHs, however, no difference was observed in menu pattern according to the physiological condition [8].

\section{Research design and methodology}

The present study being basically conceptual in nature manifests the author's own opinion and the opinion of some reputed authors. The study is mainly based on secondary data collected from websites, journals, magazines, periodicals, books, etc. The researcher also visited inmates residing in Old Age Homes $(\mathrm{OAH})$ of Purulia and her surrounding areas. Out of 200 inmates, 150 inmates were selected at random. Inmates aged 60 years and above were considered residing in $\mathrm{OAH}$ and others. Older with completely bed-ridden were excluded from the study to suit the purpose of the research. Interviews were taken through issue of structured questionnaire to the inmates. Questionnaires were prepared in the languages comprehensible to them (Bengali, Hindi and English) and contain two sections i.e. Section I contains the questions concerning demographic profile of the subjects like name, sex, religion, education, home address, etc. and Section II pertains to health, nutritional status, physical activity and dietary habits, etc. Clinical symptoms, communicable and non-communicable disease conditions were recorded. Physical activities such as walking, yoga, gardening performed by inmates were also noted. Details of dietary habits were collected from the food frequency questionnaire.

\section{Food science-its concept}

Food science is concerned with the science of nature devoted to the study of food. The Institute of Food Technologists defines food science as "the discipline in which the engineering, biological and physical sciences are used to study the nature of foods, the causes of deterioration, the principles underlying food processing and the improvement of foods for the consuming public" [9]. Food science applies science like physics, chemistry, microbiology, physiology, engineering, etc. for the study of food. Principles of biological and physical sciences are applied to study the nature of food in this discipline, the reasons of their deterioration and the principles underlying the processing and preparation of food. Food scientists also function in government regulatory agencies and academic institutions. They are directly related to the production of food products and its properties. Food science brings together multiple scientific disciplines.

\section{Nutrition-its concept}

Nutrition focuses on metabolism and metabolic pathways through which substances inside the people are transformed from one form to another. It also highlights prevention of diseases, conditions and problems with healthy diet. Likewise, nutrition identifying dietary factors such as malnutrition, food allergies, food intolerances, etc. results in certain diseases and conditions. Nutrition examines nutrients in food, use of nutrients by body and the relationship between diet, health and disease. Nutrition includes food intake, absorption, biosynthesis, catabolism and excretion [10]. A healthy diet is the preparation of food and storage methods that preserve nutrients from oxidation minimizing the risk of diseases. Nutrition leads to wastage in acute cases and the stunting of marasmus in chronic cases of malnutrition [11]. According to WHO, malnutrition refers to deficiencies, excesses or imbalances in a person's intake of energy and nutrients.

\section{Food Science-Its Importance}

Food science is a combination of various disciplines like biology, biochemistry, engineering, molecular biology and genetics, nutrition, health science and microbiology that aims at providing better understanding of food components and materials, their conversion to healthy and safe products and delivery to the consumers. Production to consumption food system is complex and our diverse, tasty, nutritious, safe and less costly food has become easily accessible. Technological advancement accelerates in feeding the growing population of the world.

\section{Nutrition-its importance}

Nutrition builds the relationship between food and its effect on an individual's health. In comparison, food science considers chemical, biological and physical properties of food in relation to manufacturing, processing and storage of food products. Nutrition and nourishment are the supply of food materials required by organisms and cells to stay alive. Nutritional science studies how the body breaks food down and how it repairs and creates cells and tissue. It also examines the response of body to food. A human body requires major types of nutrients. All nutrients do not provide energy but are still important like water and fiber. Micronutrients are important but required in small amount. A human body cannot synthesize vitamins, the essential organic compounds. 


\section{Interaction between the two disciplines}

In the 21st century, we stand on the threshold of promise for food science and nutrition science. Food science and nutrition science have worked together to reduce risk of disease in numerous ways e.g.

(i) Iodization of salt prevents goiter;

(ii) Vitamin D fortification of fluid milk eradicates rickets;

(iii) Flour enrichment virtually eliminates morbidity and mortality related to vitamin B deficiencies like pellagra and beriberi;

(iv) Fortification of flour results significant decline in the number of neural tube defects.

\section{Current State of Relationship}

Food science and nutrition science can be termed semipermeable membranes forming the boundaries of disciplines. Working together across these boundaries continues to lead to the development of healthy foods and healthier consumers. This cooperative work i.e. functional foods nourish health benefit beyond basic nutrition and impart desirable physiological effects. Such functional foods include foods fortified with vitamin D to enhance health beyond bone formation that help in reducing the risk of cardiovascular disease, hypertension, age-related macular degeneration, cancer and total mortality. Besides, functional foods increase blood levels significantly and reduce the risk of sudden death from cardiovascular disease. Nutrition examines the relationship between foods and its effect on an individual's health. It considers topics such as obesity, malnutrition, food insecurity and nutritional deficiencies. In comparison, food science considers chemical, biological and physical properties of food in relation to its manufacturing, processing and storage of food products.

\section{Future Prognostic}

Food and nutrition science have brought sound health to millions and eliminated few diet-related diseases remarkably. Nutrition science provides big opportunity to bring enhanced health benefits for consumers. Nutrigenomics focuses on identifying and understanding the molecular-level interaction between nutrients and other dietary bioactive with the genome. Nutritionists and food scientists are working together to benefit public and individual wellbeing through more understanding about the role of diet in food science creating new food products and making current products more nutritious, convenient, reliable and safe. The discipline begins with an understanding of the plants and animals-based food and ends with an understanding of the reasons for choosing certain foods.

\section{Nutrition interventions in aging and age-associated diseases}

Nutritional status of old people is associated with age-related biological and often socio-economic changes. Aging begins right from the time of birth and this process cannot be altered, but it can be regulated and monitored for better health outcome. Health outcome can be observed through social, physical and mental well-being of any individual [12]. A total of 150 older persons were considered in the study out of which females were 90 and males were 60 . The range began with $60-90$ years. The detailed age-wise distribution is exhibited in Table 1 where around $80 \%$ of the population were between 60-80 years of age. Less food intake, sedentary lifestyle and lesser energy expenditure in older age become critical risk factors for malnutrition, especially protein and micronutrients; older are particularly at risk for marginal deficiency of vitamins and trace elements. Changes in bodily functions together with malnutrition associated with advance age increase the risk of developing several age-related diseases.

Table 1: Age-wise distribution of inmates residing in $\mathrm{OAH}$.

\begin{tabular}{|c|c|c|c|c|}
\hline \multirow{2}{*}{ Age (Years) } & \multicolumn{2}{|c|}{ Male (60) } & \multicolumn{2}{c|}{ Female (90) } \\
\cline { 2 - 5 } & $\begin{array}{c}\text { Frequen- } \\
\text { cy (n) }\end{array}$ & $\%$ & $\begin{array}{c}\text { Frequency } \\
\text { (n) }\end{array}$ & $\%$ \\
\hline $60-70$ & 20 & 31.5 & 35 & 37 \\
\hline $71-80$ & 30 & 42.1 & 40 & 45.1 \\
\hline 80 above & 10 & 26.3 & 15 & 17.7 \\
\hline
\end{tabular}

Free radicals and oxidative stress are recognized as important factors in the biology of aging and age-associated degenerative diseases. Modulation of oxidative stress by calorie restriction slows aging process and decline of body functions. So, dietary components with antioxidant activity have received attention due to their potential role in modulating oxidative stress associated with aging and chronic conditions. Dietary antioxidants reduce degenerative disease like vascular dementia, cardiovascular disease and cancer, the leading causes of morbidity and mortality among the older. Supplementation of older with vitamin E enhances immune response, delays onset of Alzheimer's disease and increases resistance to oxidative injury associated with exercise. A variety of factors like improved health care and diet, vaccinations and new drugs have contributed much to the growth of older population in USA and elsewhere. Arthritis, hypertension, heart disease, hearing impairments, orthopedics impairments, cataracts, sinusitis and diabetes are the most common health problems posing difficulties for the older in carrying out daily activities. Genetics, environment and lifestyle play vital role in the rate of increase in oxidative stress and, thus, the rate of aging and age-associated diseases.

Human subjects with the highest lifespan potential have relatively great antioxidant superoxide dismutase capacity per specific metabolic rate in comparison with small mammals. Thus, eliminating free radicals formation and reducing oxidative stress and increasing antioxidant defenses reduce rate of aging and risk of chronic disease. Besides inadequate food intake and malnutrition due to income limitations, other factors including diseases, physical disability, inability to chew adequately and the intake of multiple drugs also contribute to the risk of food insufficiency among the older. Chronic conditions also increase the requirements for certain nutrients due to changes in absorption and metabolism. Energy restriction in rodent models increase longevity if it starts at any time after the maturity of animal. Dietary restriction reduces oxidative 
stress and increases endogenous levels of antioxidant enzymes. Thus practically, it is an unwise option for increasing longevity or reducing disease risk in population. Increasing antioxidant status by feeding animals with natural or synthetic antioxidants reduces oxidative stress and, thus, contributes to their longevity. Diet containing a mixture of several dietary antioxidants can extend the longevity of animals. Diet modification without drastic reduction in energy intake together with changes in lifestyle like exercising, abstaining from smoking and moderating alcohol intake along with maintaining ideal body weight are the main recommendations for reducing risk of chronic diseases and contributing to healthy aging. Supplementary intake of antioxidant vitamins such as vitamins $\mathrm{E}$ and $\mathrm{C}$ is associated with reduced risk of age-associated chronic diseases like cardiovascular disease, certain forms of cancer, cataracts and cognitive impairment which, in turn, may contribute to the longevity and growth of older population. Dietary components of foods containing antioxidant activity such as vitamin E or nonnutritive components with antioxidant activity such as polyphones in fruit and vegetables have drawn special attention due to their potential role in modulating the oxidative stress associated with aging and age-related chronic diseases. However, supplementary levels of vitamin $\mathrm{E}$ have high potential in arresting the development of cardiovascular disease through several mechanisms, improve vessel relaxation and decrease platelet aggregation. Vitamin E supplements are more effective in preventing the development of disease rather than curing the established injury in patients with cardiovascular disease. Suppression of oxidative stress and prevention of DNA damage and mutation are one of the mechanisms by which these compounds affect cancer reduction. Angiogenesis is the formation of new blood vessels from existing ones required for providing nutrients and $\mathrm{O}_{2}$ to support tumor growth.

Dietary vitamin $\mathrm{E}$ is an effective antioxidant micronutrient to boost immune function in older. Improving immune response in older results in mild incidence of infections prevailing among the older and, thus, may contribute to healthy life. Decline in cognitive function with age is another factor arresting independence and activity in the older. Increased oxidative stress and an imbalance in antioxidant status decline cognitive function with age. Impact of dietary antioxidants on prevention of vascular dementia, stroke and atherosclerosis are other mechanisms by which dietary antioxidants may reduce the risk of dementia associated with vascular dysfunction and probably Alzheimer's disease. Dietary antioxidants retard several cognitive disorders associated with neuronal diseases. Oxidative stress and decrease in the antioxidant defense system in brain neuronal cells are considered important elements in the development and progression of Alzheimer's disease. Both vitamin $\mathrm{E}$ and selegilin help in the reduction of oxidative stress to which the neuronal cell population in Alzheimer's disease is more sensitive than other neuron-degenerative diseases. Thus, dietary antioxidants and non-nutritive antioxidants containing in fruits and vegetables protect oxidative damage in neuronal tissue and prevent deterioration of the neuronal system with aging.

(Figure 1) evidence that clinical symptoms of the inmates were weakness, blurred vision, leg swelling, constipation, dizziness, joint pain, etc. Blurred vision due to cataract was the most common symptom. Other common symptoms were weakness. Walking was the only physical activity performed by the inmates; comparatively male inmates were active. Arthritis arrested the female inmates to follow physical activity. Yoga, the very important in our daily activity according to health condition and age-related factors is almost absent among the older. It is observed that cereals were consumed on daily basis. Among fruits, only banana was provided occasionally. No deep-fried items or carbonated beverages were served. However, the inmates were not consuming all the times and although milk was served, they were preferring beverages like tea and coffee due to indigestion and maltose intolerance. No proper planned menu according to disease status was followed and no individual attention to the subjects with health issues such as diabetes, hypertension, obesity, etc. was given (Table 2).

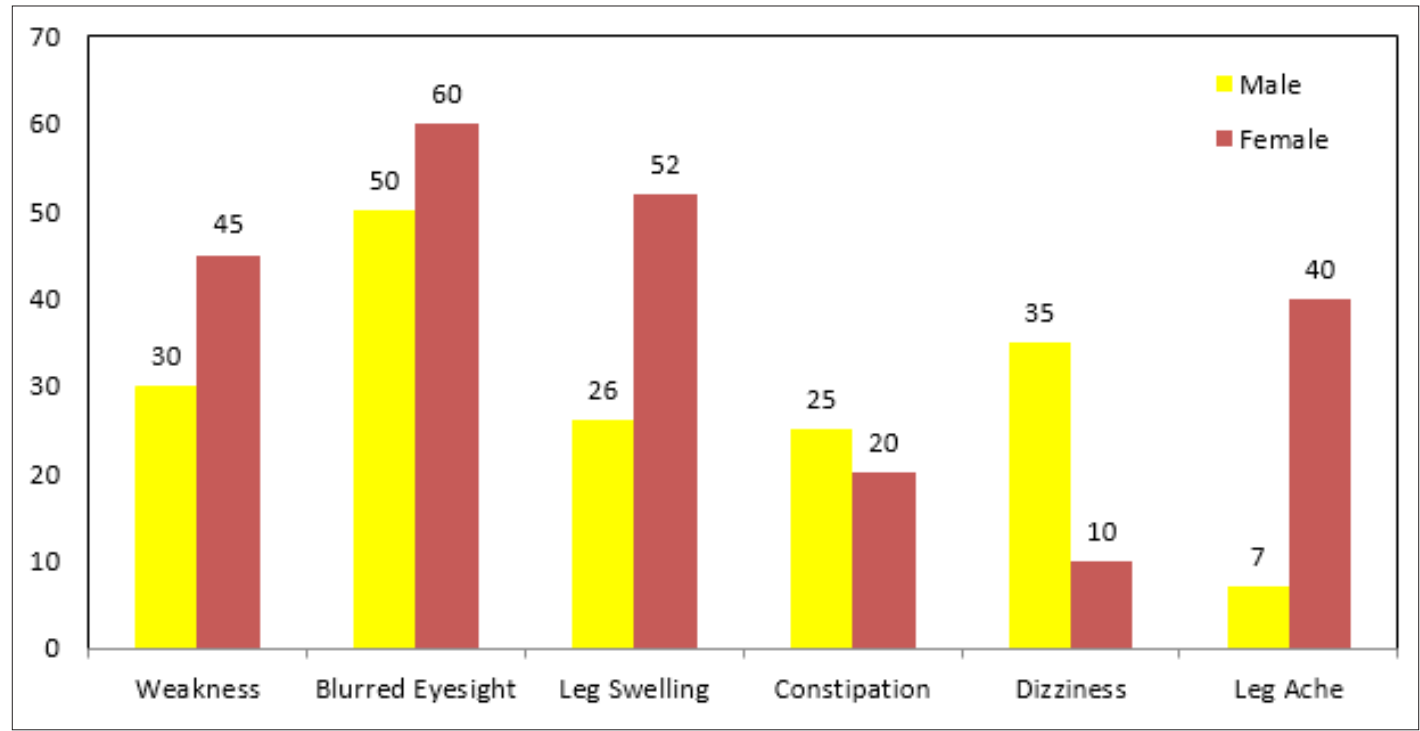

Figure 1: Clinical Sign and Symptom of Inmates Residing in OAH (\%). 
Table 2: Disease history of inmates residing in (OAH).

\begin{tabular}{|c|c|c|}
\hline Disease & Male (\%) & Female (\%) \\
\hline Diabetes Mellitus & 43 & 31 \\
\hline Hypertension & 45 & 41 \\
\hline Arthritis & 40 & 47 \\
\hline
\end{tabular}

\section{Discussion}

Apart from conceptual study, the author has also conducted study on 150 inmates residing in OAHs of Purulia (West Bengal), their original houses and their surrounding areas. The study involved the assessment of nutritional status, food frequency and disease history of the inmates. Most of the inmates were falling towards overweight and obesity due to less physical activity. Although inmates were found walking regularly, it was likely walking for less than 10 minutes having no significant impact on the weight management of the inmates. Other physical activities like yoga, gardening, etc. we're not performed by inmates due to non-availability of the provision and insufficient campus to maintain the garden. Another major reason for less physical activity was joint pain, arthritis and a few of them were lethargic. Low protein and fat status were observed in inmates. Pulses were served on regular basis; however, the quantity was not sufficient to meet their requirements and also other rich sources of protein like eggs, paneer, meat, etc., were not served. Oil added cooking is the major source of fat and no other fat-based foods were served for them. However, occasionally family, friends and outsiders visit and serve sweets or home-made food. One of the observations from food frequency is exhibited in Table 3.

Table 3: Food frequency of inmates residing in $\mathrm{OAH}$.

\begin{tabular}{|c|c|c|}
\hline Food Groups & Frequency of Consumption & $\%$ of Subjects \\
\hline \multirow{3}{*}{ Cereals } & L- occasionally & 0 \\
\hline & M- weekly 2-3times & 0 \\
\hline & H- daily & 100 \\
\hline \multirow{3}{*}{ Pulses } & L- occasionally & 0 \\
\hline & M- weekly 2-3times & 10 \\
\hline & H- daily & 90 \\
\hline \multirow{3}{*}{$\begin{array}{l}\text { M- weekly } \\
\text { 2-3times }\end{array}$} & L-occasionally & 0 Vegetables \\
\hline & 90 & \\
\hline & H- daily & 10 \\
\hline \multirow{3}{*}{$\begin{array}{l}\text { Roots \&Tu- } \\
\text { bers }\end{array}$} & L- occasionally & 10 \\
\hline & M- weekly 2-3times & 80 \\
\hline & H- daily & 10 \\
\hline \multirow{3}{*}{$\begin{array}{l}\text { Milk \& Milk } \\
\text { Products }\end{array}$} & L- occasionally & 5 \\
\hline & M- weekly 2-3times & 10 \\
\hline & H- daily & 85 \\
\hline \multirow{3}{*}{ Fruits } & L- occasionally & 80 \\
\hline & M- weekly 2-3times & 20 \\
\hline & H- daily & 0 \\
\hline
\end{tabular}

\begin{tabular}{|c|c|c|}
\hline \multirow{3}{*}{ Fried foods } & L- occasionally & 90 \\
\cline { 2 - 3 } & M- weekly 2-3times & 10 \\
\cline { 2 - 3 } & H- daily & 0 \\
\hline
\end{tabular}

\section{Suggestion}

a) Special care in inmates concerning their environmental surrounding, health profile and nutrition is required.

b) Menu should be served according to health status of the inmates.

c) Regular health check-up screening the nutritional status by trained professionals should be introduced for the inmates.

d) Inmates personally can go out for their regular health check-up and medication subject to their health conditions.

e) Nutrition or diet-related education can be imparted to inmates for taking care of their physiological conditions.

f) Periodical monitoring of the physiological conditions like diabetes, hypertension, dyslipidemia, arthritis, etc., is also suggested.

\section{Conclusion}

Contribution of dietary or supplementary antioxidants enhances life expectancy and growth of older population. Balanced diet, physical activity and abstaining from smoking together with better medical care may contribute to increase in life expectancy. Importance of micronutrients in increasing the vitality of several bodily functions such as immune, cognitive and cardiovascular among the older can be emphasized. In addition, supplementary intake of antioxidants and other micronutrients is important in preventing the onset of several age-associated chronic diseases like cardiovascular disease, cancer, dementia and infections, the major causes of morbidity and mortality among the older. Nutritional interventions being more practical and cost-effective can be implemented easily. However, separate individual diet pattern in OAHs is not possible. Change in the menu pattern and educating the inmates concerning dietary care can be emphasized for their physiological conditions. Physical activity should be their daily routine in preventing overweight and obesity. Regular monitoring and intervention can improve the health conditions of the inmates. OAH management should support proper menu and well-nourished food according to their health conditions. Minimum counseling should be specified to inmates about their preference to food.

\section{Research Scope}

Food and nutrition science in India are in infancy stage. In India, aged persons are unaware of the maintenance of proper nutritional care and importance of physical activities in their daily lives. Further studies on developing awareness by imparting nutritional education, health management in inmates residing in OAHs can be pursued. Developing nutritional awareness, promoting health care and better dietary practices especially disease specific subjects for the older are the specific areas which need deep study. 


\section{Concluding Comment}

Inmates suffering from hypertension, arthritis and diabetes mellitus should involve themselves in regular exercise. Walking is found to be the only exercise. Major observation of the study is that menu followed is not satisfying their nutritional requirements. Disease specific menu is not found to be served among the older. Regular medical check-up is also not in practice.

\section{Acknowledgement}

This paper is dedicated to ALMIGHTY GOD who bestows HIS blessings in all walks of my life.

\section{References}

1. Sharma KL (2007) Studies in Gerontology. In: Heldman, Dennis R (Eds.), IFT and the Food Science Profession, Rawat Publications, New Delhi, India, 11: 132.

2. Rajan SI, Sankara SP, Mishra US (2003) Demography of Indian aging, 2001-2051. J Aging Soc Policy 15(2-3): 11-30.

3. Jamuna D (2008) Issues of elder care and elder abuse in the Indian context. J Aging Soc Policy 15(2-3): 125-142.
4. Khole CV, Soleti A (2018) Nutritional status of elderly in the old age homes: A study in Pune city. Curr Res Nutr Food Sci Jour 6(1): 234-240.

5. Magdalena K, Patricia C, Daisy L (2008) Helping older adults meet nutritional challenges. J Nutr Elder 27(3-4): 205-220.

6. Genius SJ (2005) Nutritional transition: A determinant of global health. J Epidemiol Community Health 59(8): 615-617.

7. Payette $H(2000)$ Nutrition risk factors for institutionalization in a freeliving functionally dependent elderly population. J Clin Epidemiol 53(6): 579-587.

8. Komal C, Pallavi M, Hemangini G, Annapurna M, Hetal S (2014) Nutrition and health profile of elderly females residing in old age homes in four major cities of Gujarat. Food Sci Res J 5(2): 75-80.

9. Joint Collection Development Policy: Human Nutrition and Food (2014) US National Library of Medicine, National Institutes of Health.

10. Whitney E, Rolfes, Sharon R (2013) Understanding nutrition. $\left(13^{\text {th }}\right.$ edn), Cengage Learning, Wadsworth, USA, pp. 667-670.

11. Meydani M (2006) Nutrition interventions in aging and age-associated disease. Ann N Y Acad Sci 928: 226-235.

12. Wunderlich S (2013) The Importance of Appropriate Nutrition Assessment and Nutrition Education for Older Adults. J Nutr Food Sci $3(5)$. 